\title{
Multiple UAV Engagement Decision by Game Theory
}

\author{
Atilla Özpala*, Mehmet Önder Efe, Hayri Sever \\ Department of Computer Engineering, Hacettepe University Beytepe 06800, Ankara, Turkey. \\ * Corresponding author. Email: aozpala@hacettepe.edu.tr \\ Manuscript submitted February 5, 2017; accepted March 8, 2017. \\ doi: 10.17706/ijcee.2017. 9.1.384-392
}

\begin{abstract}
Importance of Unmanned Combat Aerial Vehicles (UCAVs) in air combat has been increasing continuously. Dangerous nature of air combat, limits of human body, high cost of pilot training and combat readiness create a requirement for using (UCAV) in the battlefield. High speed, limited time for making decision and multivariable nature of the problem are the main challenges of the autonomous vehicle development problem. Another challenge is coordination of air combat when performed by multiple fighters. In this article, we propose a decision-making method for multi UCAV air combat. In our method, each UCAV chooses the best engagement for the advantage of team instead of its own advantage and provide a real time feedback for improving the engagement decision. An incomplete information zero sum game implemented to antagonistic team pair. And a reduction method is proposed for mixed Nash Equilibrium strategies when large number of agents is involved. Promising results have been obtained in multi-air engagement scenario, and our solution is based on game theory approaches.
\end{abstract}

Key words: Engagement scenario, game theory, UAV.

\section{Introduction}

The interest to Unmanned Aerial Vehicles (UAVs) is increasing continuously. Since the application fields are very wide and diverse, the technology of UAVs is developing rapidly. One particular use is the role in battle field. UAVs can be involved in dogfight as fighters and carry weapons. Due to these capabilities, they can change the game dramatically. This paper considers how this could be done in a multi UAV engagement, which is a very important topic in defense industries.

When comparing the air power of a country, the number of pilots and their qualities are also decisive. The usage of UCAVs instead of manned aircraft come to the fore in the battle field due to reasons such as the spiritual value of human life, high cost of keeping the war pilot prepared and the maneuvering ability of the aircraft is very high but restricted with the limitations of the human body. Existing UCAVs are controlled remotely by pilots from ground. They can takeoff, land and cruise way points, but cannot fight autonomously.

Several remarkable works have been published in literature. Using Approximate Dynamic Programming (ADP), a real time autonomous one-to-one air combat method is studied and the results are tested in Realtime indoor autonomous vehicle test environment (RAVEN) [1]. Fuzzy Markov game theory is used for selecting predefined basic flight maneuvers in one-to-one engagement [2]. A game theory based solution is applied to multi UCAV engagement problem for obtaining the best response for each UCAV in each team [3]. Artificial neural networks are applied in [4] for selecting the best maneuver. An implementation of advantage function based on relative geometry is studied in [5]. Target assignment is studied in [6]-[8]. In 
[9], an optimized engagement policy performed to multiple UAVs for assigning to right enemy by using very large scale neighborhood (VLSN) search algorithm. A differential game method is implemented on a distributed formation control problem in [10]. Particle swarm optimization, ant colony optimization and game theory are applied on a cooperative air combat framework and the results are compared in [11].

In this study we focus on target mapping for two antagonistic teams of UCAVs. It is examined in case of encountering two groups of multiple airplanes in the battle field. First the superiorities between each agent in both teams are determined. Each agent is matched against the opposing groups one to one, the status of superiority between them is examined and a target is assigned to each air vehicle for the advantage of its team instead of its own advantage.

In this study, target assignment problem is not solved by analyzing the best strategy for an agent for its own advantage but the game theory is applied to determine the most suitable strategy (assignment combination) for the whole team. From this perspective, teams are the players of game and the UCAVs matching combinations to their opponents are the strategies of teams. The game is designed as a two player, zero sum game with incomplete information.

This paper is organized as follows: In the next section, a brief description of multi air engagement problem is given. Third section introduces the method for calculating air engagement superiority. Forth section elaborates the proposal of game theory implementation. Fifth section simulates a $4 \times 4$ air combat engagement. Sixth section discusses the results and the last section summarizes the concluding remarks.

\section{Problem Definition}

\subsection{Air Combat}

The operation basically consists of 3 main phases: Strategic phase includes task assignment (defense, attack) and determination of the starting positions in the battle field. Tactical phase includes target selection, engagement type determination (defense, attack) and target update. Operative phase includes basic fighter maneuver selection, primitive maneuver flow determination and aircraft control.

Since there are many political, geographical and sociological factors in the strategic level, computers are used as decision support systems and the main decision is given by humans.

Although the work is aimed at computer control at the operative level, the process is developing very rapidly and the computers are not able to make fast, effective and more successful decisions than the human brain focused on a particular task.

On the tactical level, the process is slower than the operative level, but the field of decision is expanding. It is unlikely that a target-focused human brain will detect the presence of another enemy that is superior to its enemy in a moment and change its target. However, a computer, which does not have the stress of the initial follow-up, can detect and change the other aircrafts easily. In addition, even if they are in communication, it is not possible for the pilots to accurately reflect their situation easily. Common picture can be seen at any time in the software environment and the superiority calculations can be made efficiently.

\subsection{A Tactical Level Decision: Multi UCAV Engagement}

For manned aircraft's formation flights, leader matches the opponents with the team mates the main rule is that the leader matches the opponent leader and the rest shares the others. Further to this, for manned aircraft, the most important issue is to stay alive. This means that there are two objective functions for manned aircraft; one of them is individual advantage, the other is team's advantage. Generally the individual advantage dominates team's advantage on the decision making process

When it comes to UCAVs, team's advantage is the unique objective function that makes a team one single player. Combinations of different possibilities of UCAVs engagements are strategies for relevant team. 
In a multiple UCAV engagement, there are two teams, which are friendly forces, called blue team ' $\mathrm{B}$ ', and enemy forces, called red team ' $R$ '. The teams are defined as below.

$$
\left\{B_{1}, B_{2} \ldots B_{n}\right\} \in B \text { and }\left\{R_{1}, R_{2} \ldots R_{n}\right\} \in R
$$

The processes involved in multiple air engagement are performed in fallowing steps.

- Calculate the superiorities of UCAVs to each other,

Superiorities among opponents are calculated based on variables such as situation, relative deployment, munitions loads, air craft type etc. In this study, we focus on a situational approach. This method includes weighted sum of angle, distance and speed superiorities. The superiority values are in between -1 and +1 . Positive values indicate the superiority of team $B$ and negative values indicate superiority of team $R$.

- Determine the strategies that can be played according to the calculated superiorities,

Each player can be engaged to any member of its opponent offensive or defensive role depending on their relative superiority. In this case, in a game among $\mathrm{n}$ players in each team there are $n^{n}$ possible strategies for each team. This means that we need to create a $n^{n} \times n^{n}$ strategy matrix.

- Calculate gains based on every possible strategies combination,

For each strategy pair in strategy matrix and for each player Time to Defeat (TTD) is calculated and players are eliminated in min-max sequence of TTD. It is taken into consideration if any player's defeater was eliminated before him, and infinite TTD assigned to this player. Eventually, the team, which has more remaining players than the other, is the winner of that strategy pair. The payoff is the ratio of the difference between the teams' remaining players to the initial numbers of team players.

- Choose the most appropriate strategy according to the determined strategies and achievements.

It is nearly impossible to find a solution manually because of computational complexity. In this step, we propose a "Game Theory" implementation on the strategy matrix described in the sequel.

\section{Calculating Superiority}

In dynamic air combat field, the most important superiority between agents depends on situational information. Generally, situational information contains three major parameters, namely, angle superiority, range superiority and speed superiority [7]. The situational value set is as given below.

$$
\left\{B_{x, y}, R_{x, y}, V_{b}, V_{r}, B_{\psi}, R_{\psi}\right\}
$$

\subsection{Angle Superiority}

In a one-to-one air combat scenario, the main objective is to get a position behind the opponent and bringing the target into Weapon Engagement Zone (WEZ), [12]. From this perspective, (3) calculates the angle superiority.

$$
S_{a}=\frac{|q|-|\varphi|}{180}
$$

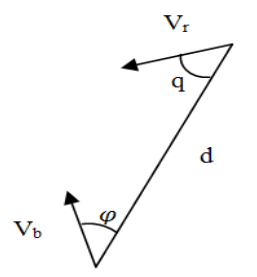

Fig. 1. Angele superiority. 


\subsection{Range Superiority}

The calculation of range superiority depends on the distance between UCAVs and also the maximum and the minimum range of the weapons loaded to UCAVs. The expression in (4) calculates the range superiority by taking account the distance and maximum, minimum range of weapons $\left(\mathrm{W}_{\mathrm{R}}\right)$.

$$
\begin{gathered}
S_{r}=e^{-\left(\frac{R-R_{0}}{\sigma}\right)^{2}} \\
R=\left(W_{R_{\max }}+W_{R_{\max }}\right) / 2, \quad \sigma=2 \times\left(W_{R_{\text {max }}}-W_{R_{\text {min }}}\right)
\end{gathered}
$$

\subsection{Speed Superiority}

Speed is the third important variable. In this study, speed means the energy of UCAV defined as below.

$$
S_{s}=1 /\left(1+e^{-6\left(\frac{V_{r}}{V_{b}}\right)+6}\right)
$$

\subsection{Total Superiority}

Total superiority is the weighted sum of predefined superiorities.

$$
S_{T}=k_{1} S_{a}+k_{2} S_{r}+k_{3} S_{S}
$$

There, $0<k_{1}, k_{2}, k_{3}<1$ and $k_{1}+k_{2}+k_{3}=1$.

\section{Multiple UCAV Engagement Decision by Game Theory}

The history of game theory dates back a long time, but the foundations of modern practices were laid by American mathematician John Forbes Nash in 1950. The four articles he published in 1950-1953 have caused the reviewing of rules known in the economy [13], [14].

Game theory, which is primarily applied in economics, mathematics, and later in biology and sociology, has been used in engineering since the early 1990s.

\subsection{Assumptions in Game}

In this game, UCAVs called as agents and two teams with same number of agents is confronting in an air battle scenario. It is assumed that;

- All agents are the same in terms of type and capability,

- The situational information of all agents are known by each agent in nearly real time,

- Every team member has the strategy information of all other agents in the same team and the strategies of the opponent team are not available.

\subsection{Preparing Superiority Matrix}

Superiority matrix is prepared using the method introduced in the previous section. Superiority is calculated for each agent pair of the B and $\mathrm{R}$ teams.

$$
\operatorname{supMat}_{T}^{i, j}=k_{1} S_{a}^{i, j}+k_{2} S_{r}^{i, j}+k_{3} S_{S}^{i, j}
$$

While a zero sum game is implemented in this study, only the highest superiority of agent pair is taken into consideration. At the beginning, both of the pairs' superiorities are calculated, and then the superior 
agent's value is assigned to the superiority matrix.

\subsection{Preparing Strategy Matrix and Game Matrix}

Superiority matrix (supMat $(n x n)$ ) indicates the superiority of each opponent pair. Agent-Opponent Matrix $\left(a o M a t_{(2 n \times n)}\right)$ is a tool for preparing strategy matrix.

$$
\text { aoMat }=[\text { supMat }- \text { supMat'] }
$$

The game matrix is produced by the strategy matrix. The gains for each strategy pair are calculated and assigned to the strategy matrix by Algorithm 1.

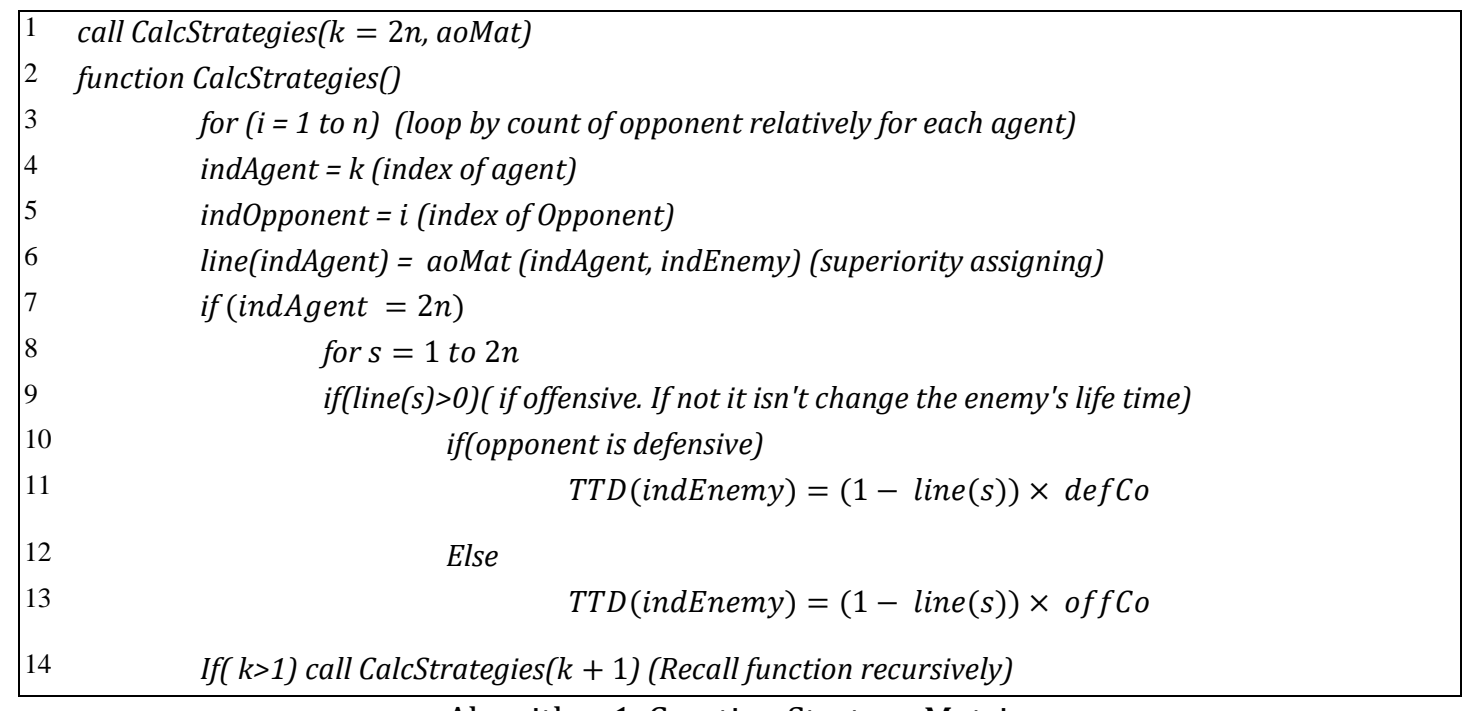

Algorithm 1. Creating Strategy Matrix.

After preparing superiority matrix, game matrix prepared by Algorithm 2.

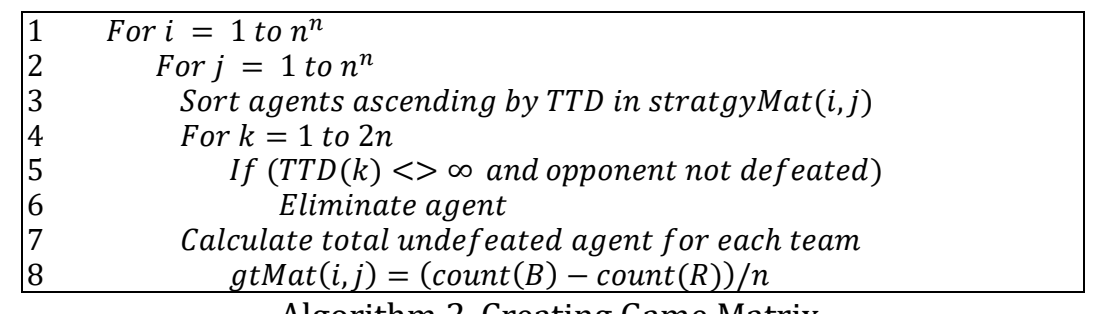

Algorithm 2. Creating Game Matrix.

The $n^{n} \times n^{n}$ game matrix obtained above is a zero sum game matrix, which means that for any strategy pair, the total gain of two teams is zero.

\subsection{Implementing Game Theory}

Game is implemented using this matrix in three steps explained as below.

- $\quad$ Find and eliminate dominated strategies

If a strategy yields a result always worse than any other strategy of the team, then this strategy is dominated and never used. Then it can be eliminated.

- $\quad$ Search dominant strategies and pure Nash Equilibria (NE)

If a strategy is always better than or equal to all other strategies of a team, then this strategy is a dominant strategy and related player only plays this strategy. Further, if a strategy is the best strategy for 
both players at its row and column respectively, it means there is a pure NE. Yet in zero sum games, this is almost impossible as one's best strategy in a row is very unlikely to be the best strategy of others in a column.

\section{- $\quad$ Calculate mixed NE}

Calculating mixed NE has a high computational complexity. In multi UCAV engagement decision scenario there is a game matrix ( gtMat $_{\mathrm{n}^{\mathrm{n}} \times \mathrm{n}^{\mathrm{n}}}$ ) which grows up exponentially. Mixed NE finds the probability coefficients for each strategy. If there are $\mathrm{n}^{\mathrm{n}}$ strategies, in a balanced distribution each strategy has a $1 / \mathrm{n}^{\mathrm{n}}$ probability. The probabilities below a defined threshold $\varepsilon$ are not significant. If we underestimate the probabilities below $\varepsilon$, even if the strategies are perfectly balanced; maximum strategy count is $1 / \varepsilon$. The expression for significant strategies can be given as below.

$$
S_{R}=\frac{1}{\varepsilon}-\frac{1}{\varepsilon} / n
$$

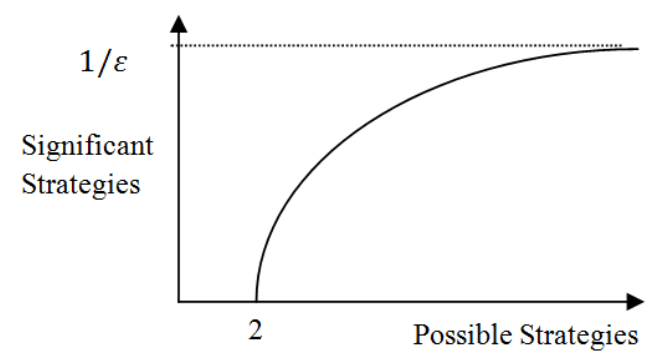

Fig. 2. Limit of significant strategies count.

Fig. 2 is the graphical representation of significant strategy count, depending on possible strategies. The count of significant strategies limited at $1 / \varepsilon$ and for approximate to this value $\frac{1}{\varepsilon} / n$ subtracted from $1 / \varepsilon . n$ is the number of agent in each team. We need to eliminate unreasonable strategies by reducing the game matrix. For this reason, first, the sum of each column and row are calculated and getting the significant strategies according to the expression shown above.

Algorithm 3 is used for decreasing the size of the problem before calculating the mixed NE.

$$
\begin{array}{|cr}
\hline 1 & B_{\text {sum }}^{k}=\sum_{i=1}^{n} \operatorname{gtMat}_{(k, i)} \\
2 & R_{\text {sum }}^{k}=\sum_{i=1}^{n} \operatorname{gtMat}_{(i, k)} \\
3 & \text { Get max } 1 / \varepsilon \text { terms from } B_{\text {sum }}^{k} \\
4 & \text { Get min } 1 / \varepsilon \text { terms from } R_{\text {sum }}^{k}
\end{array}
$$

Algorithm 3. Strategy Elimination.

\section{Game Simulation}

In our example game, there are two teams each having four players. ' $\mathrm{B}$ ' and ' $\mathrm{R}$ ' are friendly and enemy forces respectively. The initial positions of agents in a 25x25 sized area are shown in Table 1.

Table 1. Initial Positions

\begin{tabular}{|c|c|c|c|c|c|c|c|c|}
\hline & $\mathbf{B}_{\mathbf{1}}$ & $\mathbf{B}_{\mathbf{2}}$ & $\mathbf{B}_{\mathbf{3}}$ & $\mathbf{B}_{\mathbf{4}}$ & $\mathbf{R}_{\mathbf{1}}$ & $\mathbf{R}_{\mathbf{2}}$ & $\mathbf{R}_{\mathbf{3}}$ & $\mathbf{R}_{\mathbf{4}}$ \\
\hline $\mathbf{x}$ & 2 & 4 & 3 & 5 & 23 & 21 & 21 & 23 \\
\hline $\mathbf{y}$ & 3 & 10 & 16 & 22 & 3 & 7 & 15 & 22 \\
\hline $\boldsymbol{\psi}$ & 105 & 60 & 75 & 120 & -135 & -75 & -120 & -60 \\
\hline
\end{tabular}


The superiorities between two teams' players are shown in Table 2. Positive values are the blue team's advantage and negatives are the red team's advantage.

Table 2. Superiority Matrix

\begin{tabular}{|c|r|r|r|r|}
\hline $\mathbf{B} / \mathbf{R}$ & $\mathbf{R}_{\mathbf{1}}$ & \multicolumn{1}{|c|}{$\mathbf{R}_{\mathbf{2}}$} & \multicolumn{1}{c|}{$\mathbf{R}_{\mathbf{3}}$} & $\mathbf{R}_{\mathbf{4}}$ \\
\hline $\mathbf{B}_{\mathbf{1}}$ & -0.43 & 0 & -0.47 & 0.33 \\
\hline $\mathbf{B}_{\mathbf{2}}$ & -0.38 & -0.36 & 0 & 0.52 \\
\hline $\mathbf{B}_{\mathbf{3}}$ & 0.33 & -0.43 & -0.39 & 0.39 \\
\hline $\mathbf{B}_{\mathbf{4}}$ & 0.54 & 0.37 & 0.5 & 0 \\
\hline
\end{tabular}

The Strategy Matrix $\left(n^{2 n} \times 2 n \times 4\right)$ has been created by Algorithm 1 . The three dimensions of matrix are;

- First dimension $\left(n^{2 n}\right)$ is the total count of strategies.

- Second dimension ( $2 n)$ is the total count of agents,

- $\quad$ Third dimension (4);

○ Index of opponent,

- Superiority value between the agent and opponent,

- TTD for relevant agent,

- Opponent that engaged to agent with the lowest TTD.

Game Matrix is created by Algorithm 2 is used as an input quantity for this algorithm. Strategy matrix produces all possible strategies for both teams and TTD for each agent. TTD is the expected life time of an agent.

$$
\operatorname{TTD}_{A_{i}}=\min _{k=1 \text { to } n}\left(\left(1-\text { comSupMat }_{A_{i}, o_{k}}\right) \times t\right)
$$

Having these computations completed, game matrix is created by Algorithm 2. The strategy matrix is an important parameter for this algorithm. The size of the game matrix is $n^{n} \times n^{n}$ (strategies of Blue team $\times$ strategies of Red team).

Dominant strategies are searched first. In this example there are no dominant strategies. After dominant strategies, pure NEs are searched even there is no pure NE and dominated strategies are eliminated after. The matrix is very large for finding a mixed NE. It is not only computationally complex, but also there are many meaningless strategies. The size of matrix is decreased by Algorithm 3 In this example, $\varepsilon=0.05$ and $1 / \varepsilon=20$ is the maksimum count of reasonable strategies. After eliminating the dominated strategies, finally a matrix of size $11 \times 10$ is obtained and this matrix contains all reasonable strategies. We call this as purified game matrix and tabulate its content in Table 3.

Table 3. Purified Game Matrix

\begin{tabular}{|c|c|c|c|c|c|c|c|c|c|c|}
\hline & $\mathbf{2 6}$ & $\mathbf{2 8}$ & $\mathbf{3 4}$ & $\mathbf{4 2}$ & $\mathbf{4 6}$ & $\mathbf{9 8}$ & $\mathbf{9 9}$ & $\mathbf{1 0 2}$ & $\mathbf{1 0 6}$ & $\mathbf{1 6 2}$ \\
\hline $\mathbf{3}$ & -0.25 & -0.25 & -0.25 & -0.25 & -0.25 & -0.25 & 0 & -0.25 & -0.25 & -0.25 \\
\hline $\mathbf{5 0}$ & 0 & 0 & -0.5 & -0.25 & -0.25 & -0.25 & 0 & 0 & 0 & -0.5 \\
\hline $\mathbf{5 1}$ & 0 & 0 & -0.25 & 0 & -0.25 & -0.25 & 0.25 & 0 & 0 & -0.25 \\
\hline $\mathbf{1 1 5}$ & 0 & 0 & -0.25 & 0 & -0.25 & -0.25 & 0.25 & 0 & 0 & -0.25 \\
\hline $\mathbf{1 7 9}$ & 0 & 0 & -0.25 & 0 & -0.25 & -0.25 & 0.25 & 0 & 0 & -0.25 \\
\hline $\mathbf{1 8 5}$ & 0.25 & 0.25 & 0 & 0 & 0.25 & 0.25 & 0 & 0.25 & 0.25 & 0 \\
\hline $\mathbf{1 9 4}$ & -0.25 & -0.25 & -0.5 & -0.5 & 0 & 0 & -0.25 & -0.25 & -0.25 & -0.5 \\
\hline $\mathbf{1 9 5}$ & -0.25 & -0.25 & 0 & 0 & 0 & 0 & 0.25 & -0.25 & -0.25 & 0 \\
\hline $\mathbf{2 4 2}$ & -0.25 & -0.25 & -0.5 & -0.25 & 0 & 0 & -0.25 & -0.25 & -0.25 & -0.5 \\
\hline $\mathbf{2 4 3}$ & -0.25 & -0.25 & 0 & 0 & 0 & 0 & 0.25 & -0.25 & -0.25 & 0 \\
\hline $\mathbf{2 4 7}$ & 0 & -0.25 & 0 & 0 & 0 & 0.25 & 0.25 & 0 & -0.25 & 0 \\
\hline
\end{tabular}

After running the mixed strategy on matrix shown in Table 3, the calculated mixed solution for red team 
is shown in Table 4 and the mixed solution for red team is shown in Table 5.

Table 4. Mixed NE for Blue Team

\begin{tabular}{|l|l|l|l|l|l|l|}
\hline Seq & B1 & B2 & B3 & B4 & Probability & $\begin{array}{l}\text { Winning } \\
\text { Percentage }\end{array}$ \\
\hline 51 & 1 & 4 & 1 & 3 & 0.167 & 47.66 \\
\hline 185 & 3 & 4 & 3 & 1 & 0.333 & 28.52 \\
\hline 195 & 4 & 1 & 1 & 3 & 0.203 & 45.31 \\
\hline 243 & 4 & 4 & 1 & 3 & 0.297 & 45.7 \\
\hline
\end{tabular}

Table 5. Mixed NE for Red Team

\begin{tabular}{|c|c|c|c|c|c|c|}
\hline Seq & R1 & R2 & R3 & R4 & Probability & $\begin{array}{c}\text { Winning } \\
\text { Percentage }\end{array}$ \\
\hline 26 & 1 & 2 & 3 & 2 & 0.333 & -27.2 \\
\hline 42 & 1 & 3 & 3 & 2 & 0.062 & -18.8 \\
\hline 46 & 1 & 3 & 4 & 2 & 0.105 & -18.8 \\
\hline 98 & 2 & 3 & 1 & 2 & 0.5 & -40.1 \\
\hline
\end{tabular}

The approach defined in this paper therefore proposes a reasonable engagement strategy for multiple UAV combat missions.

\section{Conclusion and Future Work}

The method proposed in this study gives more importance to team's advantage than the UCAVs own advantage. First the superiority is calculated according to UCAVs situational information. Payoffs and TTDs are calculated by using superiority matrix for each strategy. We have a strictly cooperative game and there are only two teams playing the game. Every team has a number of agents. A game matrix composed of all possible strategies is constructed. Due to its very large size, a reduction approach is proposed and implemented. The most reasonable $1 / \varepsilon$ strategy is selected and mixed strategies are sought on this matrix $((1 / \varepsilon) \times(1 / \varepsilon))$. This means the exponential strategy count reduce to scalar values. The approach is tested on a numerical case and its effectiveness is demonstrated.

In the future, we aim at implementing this approach on a dynamically changing environment and agent numbers.

\section{References}

[1] McGrew, J., How, J., Bush, L., Williams, B., \& Roy, N. (2010). Air combat strategy using approximate dynamic programming. Journal of Guidance, Control and Dynamics, 33(5), 1641-1654.

[2] An, X., Yingxin, K., Lei, Y., Baowei, X., \& Yue, L. (2011). Engagement maneuvering strategy of air combat based on fuzzy markov game theory. Proceedings of 2011 IEEE 2nd International Conference on Computing, Control and Industrial Engineering (CCIE), 2.

[3] Chen, X., \& Mingming, Z. (2012). The decision method research on air combat game based on uncertain interval information. Proceedings of 2012 Fifth International Symposium on Computational Intelligence and Design (ISCID), 1.

[4] Teck-Hou, T., Ah-Hwee, T., Ah-Hwee, T., \& Yeo, A. (2012). Self-organizing neural networks for learning air combat maneuvers. Proceedings of the 2012 International Joint Conference on Neural Networks (IJCNN).

[5] Karl, M., Efe, M. Ö., \& Sever, H. (2015). Close range one to one air combat maneuvering for autonomous uav, 8. Proceedings of Ankara International Aerospace Conference, Turkey: Ankara.

[6] Li, F., Qi, W., Jin, X., Yuandong, Z., \& Kun, Z. (2012). Target assignment and sorting for multi-target attack in Multi-aircraft coordinated based on RBF. Proceedings of Control and Decision Conference (CCDC).

[7] Xia, C., \& Yongxin, H. (2012). Study on the task assignment for Multi-UCAV in air-combat. Proceedings of 2012 Fifth International Symposium on Computational Intelligence and Design (ISCID), 1.

[8] Chen, H., \& Zhang, K. (2012). Target threat assessment based on genetic neural network. Proceedings of 2012 International Conference on Industrial Control and Electronics Engineering (ICICEE).

[9] Lv-wei, N. Xiao-guang, G., \& Bo, L. (2011). Target assignment for multi-fighters coordinated air combat based on VLSN, signal processing. Proceedings of 2011 IEEE International Conference on 
Communications and Computing (ICSPCC).

[10] Lin, W. (May 2014). Distributed UAV formation control using differential game approach. Aerospace Science and Technology, 35, 54-62.

[11] Duan, H., Wei, X., \& Dong, Z. (Nov. 2013). Multiple UCAVs cooperative air combat simulation platform based on PSO, ACO, and game theory. IEEE Aerospace and Electronic Systems Magazine, 11, 12-19.

[12] Shaw, R. (1985). Fighter Combat Tactics and Maneuvering, Naval Institute Press Maryland: Annapolis.

[13] Nash, J. (1950). Equilibrium points in n-person games. The National Academy of Sciences, 36(1), 48-49.

[14] Nash, J. (1951). Non-Cooperative Games. The Annals of Mathematics, 54(2), 286-295.

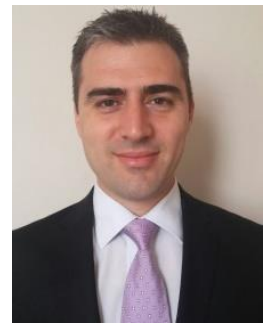

Atilla Özpala received the B.S. and M.S. degrees in computer engineering from Sakarya University, Turkey in 2003 and 2007, respectively. He is currently a doctoral researcher with the Department of Computer Engineering, Hacettepe University, Turkey. His research interests include computational game theory, machine learning and UAV control.

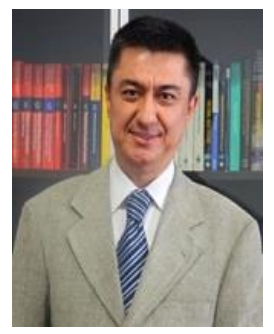

Mehmet Önder Efe received the B.S. degree from İstanbul Technical University, Electronics and Communication Engineering Department. He has completed his master's degree in Boğaziçi University System and Control Engineering Department and his Ph.D. in Electrical-Electronics Engineering Department. Prof. Efe currently works as a professor at Hacettepe University Department of Computer Engineering. Prof. Efe is the chairman of the Department, chairman of the Hardware Department, and the founder of the Autonomous Systems Research Laboratory. Prof. Efe is an assistant editor in some magazines with prestige in the world and is a member of the board of the Turkish National Committee for Automatic Control.

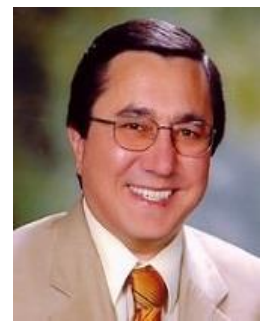

Hayri Sever received the B.S, M.S. Ph.D. degrees in computer engineering from Hacettepe University in 1986, University of Maine in 1991 and University of Louisiana respectively in 1995. Prof. Dr. Hayri Sever is the head of Department of Software Engineering at Hacettepe University Computer Engineering Department. The fields of multi-media information access (and filtering) models, vertical search engines, data and web mining, voice analysis, semantic analysis, geographic information systems and uncertainty reasoning approach areas constitute the active research topics of Dr. Sever. 